\title{
Estimation of GPS Position Using Iterative Least Squares and Extended Kalman Filter
}

\author{
Doma M. I. ${ }^{1}$ and Sedeek A. T. ${ }^{2}$ \\ 1. Civil Engineering Department, Faculty of Engineering, Menoufia University, Egypt \\ 2. Civil Engineering Department, Faculty of Engineering, Delta University, Egypt
}

\begin{abstract}
Global Positioning System (GPS) is being used in aviation, nautical navigation and the orientation ashore. Further, it is used in land surveying and other applications where the determination of the exact position is required. Although GPS is known as a precise positioning system, there are several error sources which are categorized into three main groups including errors related to satellites, propagation and GPS receivers. In this paper we focus on the estimation of the receiver coordinates of a fixed point based on pseudorange measurements of a single GPS receiver. The estimation of the unknowns is achieved by introducing an Extended Kalman Filter (EKF) and Iterative Least Square (ILS) that processes. Kalman filter is the method most often used nowadays. It is based on some assumptions and if all the assumptions are met it can offer optimal estimation and prediction. The EKF not only works well in practice, but also it is theoretically attractive because it has been shown that it is the filter that minimizes the variance of the estimation mean square error.
\end{abstract}

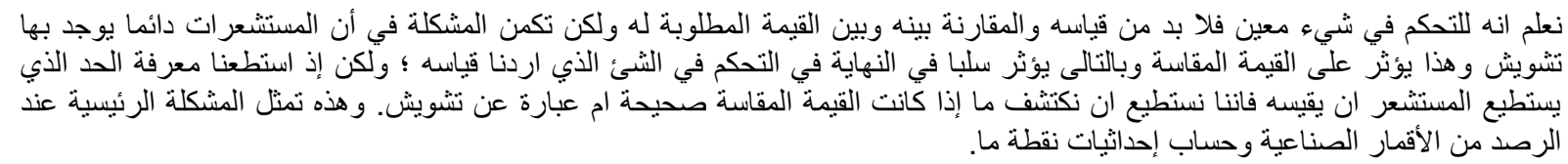

وتعتبر طريقة Iterative least squares الطريقة الرئيسية لإز الة مثل هذا التشويش وحساب موقع المستقبل ومع ظهور

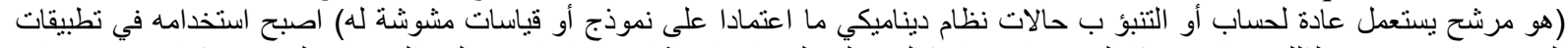

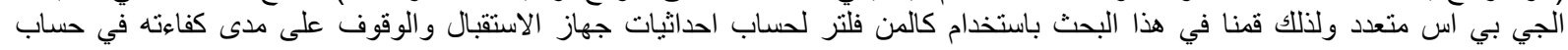

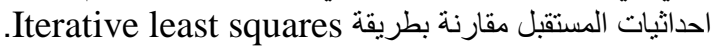

\section{INTRODUCTION}

While the compass gave the navigators knowledge of the general direction they were traveling, it did not give them their current location on the Earth's surface. One of the earliest navigation tools that allowed for a general knowledge of the current position was the astrolabe.

The astrolabe determined latitude by determining the altitude of the sun and stars. Another commonly used navigation tool for latitude determination was the sextant. The sextant was capable of providing more accurate latitude measurements by determining the altitude between the horizon and celestial bodies (Battin, 1999).

The Navy Navigation Satellite System (NNSS), also known as TRANSIT, was the first operational satellite system launched in 1972. The NNSS allowed for position determination based on satellites orbiting at an altitude of 600 nautical miles. Doppler shift was measured in a frequency constantly transmitted from the satellites. Aircraft applications of the system proved not useful since corrections had to be made based on vehicle velocity. In addition, the number of satellites were limited to five worldwide, resulting in signal blackouts that would last as long as 100 minutes (Spilker et al, 1996). In 1973, the idea of a satellite constellation capable of providing a user with an accurate position anywhere on the surface of the Earth began to become a reality.

An important class of theoretical and practical problems in navigation system is a statistical nature. Such problems are: Prediction of random signals; separation of random signals from random noise and detection of signal of known form (pluses, sinusoids) in the presence of random noise. A widely used tool in order to get an optimal, in the sense of minimizing the mean squared error, estimate of the state of a system is iterative least square and Kalman filter (Hu et al. 2003)

In this paper, we are interested in estimating the receiver coordinates, combining all the information about noise and bias error sources, 
using iterative least square and extended Kalman filter. To show the performance of each method in estimating receiver position.

\section{Navigation System and GPS Positioning}

The navigation system of satellites that makes up the space segment of GPS consists of 24 satellites allocated in six orbital planes. Each satellite transmits two carrier signals on the L1 (1575.42 MHz) and L2 (1227.6 MHz) frequencies that contain the ephemeris data for the determination of the position of the satellites. When the satellite position is known, an authorized user can receive the satellite's transmitted signals and determine the signal propagation time. By using this information, each receiver will be able to compute its ranges to the satellites and correct its clock. The actual measurement is called pseudorange $\mathbf{P}^{\mathbf{k}}$ because of the offset of the receiver from true GPS time (see Fig. 1). A minimum of four simultaneous pseudoranges are necessary for the accurate determination of the receiver position $(\mathrm{X}, \mathrm{Y}, \mathrm{Z})$ and the clock bias (dt) (Leick, 1995; HofmannWellenhof,1994) .

The whole GPS positioning procedure includes three tasks: acquisition, tracking and positioning (Borre et al., 2007). The acquisition tries to find satellites and to get their positions. It gives rough estimates of signal parameters. Tracking keeps track of these parameters as the signal properties change over time. After tracking, the navigation data can be extracted and pseudoranges (measured distance from satellites to GPS receiver) can be computed. The final task of the receiver is to compute the user position.

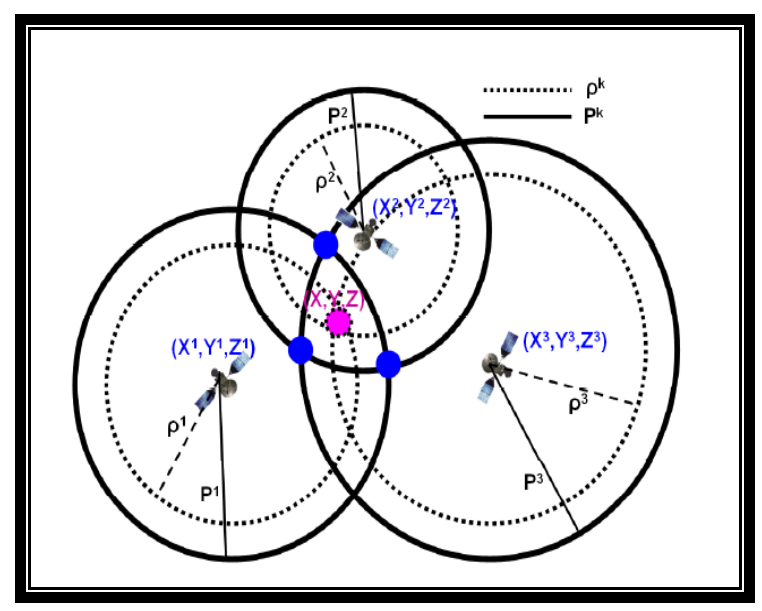

Figure 1. Observed pseudoranges $\mathrm{P}^{\mathrm{k}}$ and geometrical pseudoranges $\rho^{\mathbf{k}}$

The basic observation equation for the pseudorange $\mathrm{P}^{\mathrm{K}}$ is (He and Bilgic, 2011):

$$
\mathbf{P}^{\mathbf{K}}=\rho^{\mathbf{k}}+\mathbf{c}\left(\mathbf{d t}-\mathbf{d t ^ { k }}\right)+\mathbf{T}^{\mathbf{k}}+\ell^{\mathbf{k}}+\mathbf{e}^{\mathbf{k}}
$$

$\rho^{\mathbf{k}}$ is the geometrical range between satellite $\mathrm{k}$ and receiver, which can be computed as:

$$
\rho^{k}=\sqrt{\left(x^{k}-x\right)^{2}+\left(y^{k}-y\right)^{2}+\left(z^{k}-z\right)^{2}}
$$

Where $(\mathrm{x}, \mathrm{y}, \mathrm{z})$ is the position of receiver $\mathrm{dt}$ denotes the receiver clock offset and $\mathrm{dt}^{\mathrm{k}}$ is the satellite clock offset. From the ephemerids, which also include information on the satellite clock offset $\mathrm{dt}^{\mathrm{k}}$, the position of the satellite $\left(\mathrm{x}^{\mathrm{k}}, \mathrm{y}^{\mathrm{k}}, \mathrm{z}^{\mathrm{k}}\right)$ can be computed. $\mathrm{T}^{\mathrm{k}}$ is the tropospheric error and $\ell^{\mathbf{k}}$ is the ionospheric error. These two errors are computed from a priori models, whose coefficients are part of the broadcast ephemerids. $\mathrm{e}^{\mathrm{k}}$ is the observation error of the pseudorange. Therefore, Eq. (1) contains four unknowns $x, y, z$ and dt. The error terms are minimized.

\section{Iterative Least Square Method}

The most commonly used algorithm for position computation from pseudoranges is based on the Least Square (LS) method. This method is used to find the receiver position from pseudoranges to four or more satellites.

Equation (2) is nonlinear with respect to the receiver position $(\mathrm{x}, \mathrm{y}, \mathrm{z})$, so the equation is linearized before using the LS method. The nonlinear term in Eq. (2) is linearized:

$$
\mathbf{f}(x, y, z)=\sqrt{\left(x^{k}-x\right)^{2}+\left(y^{k}-y\right)^{2}+\left(z^{k}-z\right)^{2}}
$$

Starting from an initial position for the receiver $(\mathrm{x}, \mathrm{y}, \mathrm{z})$, the position estimate is improved iteratively. The center of the Earth $(0,0,0)$ can be chosen as the initial point, if no a-prioriinformation.

The range computed from the satellite position $\left(\mathrm{x}^{\mathrm{k}}, \mathrm{y}^{\mathrm{k}}, \mathrm{z}^{\mathrm{k}}\right)$ to the approximate receiver position $\left(\mathrm{x}_{\mathrm{itr}}, \mathrm{y}_{\mathrm{itr}}, \mathrm{z}_{\mathrm{itr}}\right)$, so the first-order linearized observation equation becomes

$$
\begin{aligned}
& p_{i t r}^{k}=\rho_{i t r}^{k}-\frac{x^{k}-x_{i t r}}{\rho_{i t r}^{k}} \Delta x_{i t r}-\frac{y^{k}-y_{i t r}}{\rho_{i t r}^{k}} \Delta y_{i t r} \\
& -\frac{z^{k}-z_{i t r}}{\rho_{i t r}^{k}} \Delta z_{i t r}+c\left(d_{i t r}-d t^{k}\right)+T_{i t r}^{k}+\ell_{i t r}^{k}+e_{i t r}^{k}
\end{aligned}
$$

Where itr be the number of the iterations, $\mathrm{dt}_{\mathrm{itr}}$ is the estimated clock error at the receiver. Rearranging Eq. (4), it can be written as: 


$$
\left(-\frac{x^{k}-x_{i t r}}{\rho_{i t r}^{k}}-\frac{x^{k}-x_{i t r}}{\rho_{i t r}^{k}}-\frac{x^{k}-x_{i t r}}{\rho_{i t r}^{k}} c\left(\begin{array}{c}
\Delta x_{i t r} \\
\Delta y_{i t r} \\
\Delta z_{i t r} \\
d t_{i}
\end{array}\right)\right.
$$$$
=\mathbf{P}_{i t r}^{k}-\rho_{i t r}^{k}+c^{k} t^{k}-T_{i t r}^{k}-\ell_{i t r}^{k}-\mathbf{e}_{i t r}^{k}
$$

A unique solution can not be found from a single equation. Therefore $\mathrm{m} \geq 4$ satellites are required to form a system of linear equations (usually $m \geq 6$ satellites are available).

$$
\text { Let } \mathbf{b}_{\mathrm{itr}}^{\mathrm{k}}=\mathrm{P}_{\mathrm{itr}}^{\mathrm{k}}-\rho_{\mathrm{itr}}^{\mathrm{k}}+\mathrm{cdt}^{\mathrm{k}}-\mathrm{T}_{\mathrm{itr}}^{\mathrm{k}}-\ell_{\mathrm{itr}}^{\mathrm{k}}-\mathrm{e}_{\mathrm{itr}}^{\mathrm{k}} \text {. }
$$

Then we obtain the LS problem:

$$
\min _{\mathrm{x}_{\text {itr }}}\left\|\mathbf{A}_{\text {itr }} \mathbf{x}_{\text {itr }}-\mathbf{b}_{\text {itr }}\right\|
$$

Where:

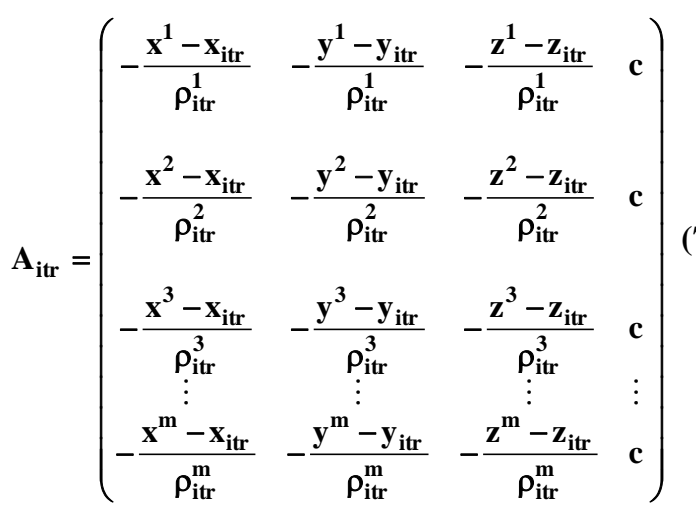

In each iteration of the positioning algorithm a LS problem must be computed. Since the pseudoranges are subject to measurement errors and the convergence (number of required iterations) of the algorithm depends on the accuracy of these LS solutions, it is worthwhile to investigate the use of an iterative LS solver and the trade-off between the number of iterations of the positioning method and the number of iterations of the iterative LS solver (He and Bilgic, 2011).

\section{Extended Kalman Filter (EKF)}

In 1960, R.E. Kalman published his famous paper on a recursive predictive filter that is based on the use of state space techniques and recursive algorithms and therewith he revolutionized the field of estimation (Kalman, 1960). Since that time, due in large part to advances in digital computing, the Kalman filter has been the subject of extensive research and application, particularly in the area of autonomous or assisted navigation.

$\mathrm{KF}$ is an optimal estimator which can result in an optimum estimation of a system state using state space principle and system error modeling. $\mathrm{KF}$ is a linear, unbiased and recursive algorithm that optimally estimates the unknown state of a dynamic system from noisy data taken at discrete real time intervals by minimizing the mean-squared error. The most important feature of $\mathrm{KF}$ is that it is recursive, hence it does not require storage of all past observations; the KF algorithm is ideally suited to dealing with complex estimation problem (Simon, 2006).

Thus, the Kalman filter consists of two steps: The prediction and The correction. In the first step, the state is predicted with the dynamic model. In the second step it is corrected with the observation model, so that the error covariance of the estimator is minimized. In this sense it is an optimal estimator (Xu, 1996)

In practice, the $\mathrm{KF}$ attempts to minimize the variance of the estimation errors and provides optimal result if perfect a priori knowledge of both the process noise and measurement noise are Gaussian white processes and the noise statistics for both disturbances are completely known. To obtain good estimation solutions using the Extended Kalman Filter (EKF) approach, the designers are required to have good knowledge on both dynamic process (plant dynamics, using an estimated internal model of the dynamics of the system) and measurement models, in addition to the assumption that both the process and measurement are corrupted by zero mean white noises (Sarras et al. 2010).

The extended Kalman filter is employed when the process model and/or the measurement model are represented by a nonlinear equation. In the point positioning problem only the measurement equation is nonlinear so, the main purpose is to find the linearized equations needed for the filter implementation.

To achieve recursive equations of EKF, we start with state and measurement equations (Chaer et al., 1997):

Where:

$$
\begin{aligned}
\mathrm{X}_{\mathrm{k}+1} & =\phi_{\mathrm{k}} \mathrm{X}_{\mathrm{k}}+\mathrm{W}_{\mathrm{k}} \\
\mathbf{Z}_{\mathbf{k}} & =\mathbf{A}_{\mathbf{k}} \mathbf{X}_{\mathbf{k}}+\mathbf{V}_{\mathbf{k}}
\end{aligned}
$$

$\mathbf{X}_{\mathbf{k}}:$ is $\mathrm{n} \times 1$ state vector at time $t_{k}$,

$\phi_{\mathbf{k}}$ : is the $\mathrm{n} \times \mathrm{n}$ state transition matrix from $\mathrm{X}_{\mathrm{k}}$ to $\mathrm{X}_{\mathrm{k}+1}$,

$\mathbf{W}_{\mathbf{k}}$ : is $\mathrm{n} \times 1$ process error vector,

$\mathbf{Z}_{\mathbf{k}}$ : is the $\mathrm{m} \times 1$ measurement vector at time $t_{k}$,

$\mathbf{A}_{\mathbf{k}}:$ is the $\mathrm{m} \times \mathrm{n}$ matrix which creates an ideal relation (without any noises) between measurement and 
$t_{k}$ and $\mathbf{V}_{\mathbf{k}}$ is the $\mathrm{m} \times 1$ error vector. The EKF recursive equations are:

$$
\mathrm{K}_{\mathrm{k}}=\mathrm{P}_{\mathrm{k}}^{-} \mathrm{A}_{\mathrm{k}}^{\mathrm{T}}\left(\mathrm{A}_{\mathrm{k}} \mathrm{P}_{\mathrm{k}}^{-} \mathrm{A}_{\mathrm{k}}^{\mathrm{T}}+\mathrm{R}_{\mathrm{k}}\right)^{-1}
$$

Where

$$
\begin{aligned}
& \mathbf{K}_{\mathbf{k}} \text { is called Kalman gain and } \\
& \mathbf{R}_{\mathbf{k}} \text { is the discrete measurement } \\
& \text { covariance matrix and is diagonal } \\
& \text { due to uncorrelated measurements. }
\end{aligned}
$$

The estimation process is updated as:

$$
\hat{\mathbf{X}}_{\mathbf{k}}=\hat{\mathbf{X}}_{\mathbf{k}}^{-}+\mathbf{K}_{\mathbf{k}}\left(\mathbf{Z}_{\mathbf{k}}-\mathbf{A} \hat{\mathbf{X}}_{\mathbf{k}}^{-}\right)
$$

Where “^” and "-" denote estimated state and prior to measurement incorporation, respectively.

By calculating the corresponding covariance matrix using optimum estimation, we will have:

$$
\mathbf{P}_{\mathrm{k}}=\left(\mathbf{I}-\mathbf{K}_{\mathrm{k}} \mathbf{A}_{\mathrm{k}}\right) \mathbf{P}_{\mathrm{k}}^{-}
$$

Consequently, optimum state estimation and covariance matrix are resulted for the next time step.

$$
\begin{aligned}
& \mathbf{X}_{\mathrm{k}+1}^{-}=\phi_{\mathrm{k}} \hat{\mathbf{X}}_{\mathrm{k}} \\
& \mathbf{P}_{\mathrm{k}+1}^{-}=\phi_{\mathrm{k}} \mathbf{P}_{\mathrm{k}} \boldsymbol{\phi}_{\mathrm{k}}^{\mathrm{T}}+\mathbf{Q}_{\mathrm{k}}
\end{aligned}
$$

\section{Results and Analysis}

For GPS positioning, a Pro Mark 100-L1 was used to capture the raw GPS data, which is single signal data being delivered by the GPS satellite network (See photo 1)

The obtained raw GPS data at each measurement point includes information of $m=8$ satellites with matched pseudoranges. 25 raw GPS data records are gained with 30 second as an interval. Afterwards position calculation is done for GPS positioning using ILS and EKF.

In the present study, both observation and navigation data were in Rinex format, the data processing program is written in MATLAB program and consists of three sessions:

1. Reading the observation file and manipulating its data.

2. Reading the navigation file and manipulating its data.

3. Estimating the receiver coordinates using iterative least squares method and the extended Kalman filter.

Also, MATLAB code was needed the positions of satellites to compute the estimated coordinates, so, in present work, the positions of satellites were estimated using the data of Analysis Centers (ACs).

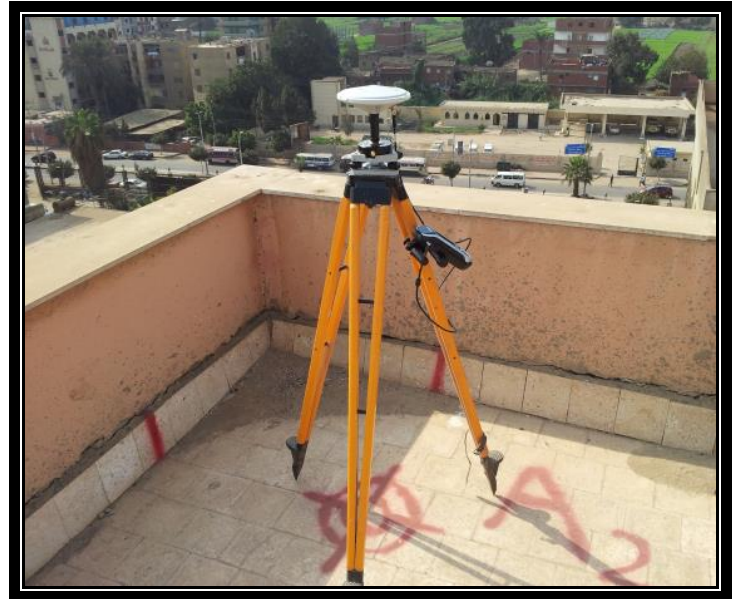

Photo 1. GPS instrument "Pro Mark 100 - L1 model ASH-660 L1"

The IGS, formerly the International GPS Service, data web site was used in satellite position computations are given at $900 \mathrm{sec}(15 \mathrm{~min})$ time steps, in current study we interpolate the position of each satellite to provide a reasonable satellite data at equal time steps of ( 30 sec.). The IGS is committed to providing the highest quality data and products as the standard for Global Navigation Satellite Systems (GNSS) in support of Earth science research, multidisciplinary applications, and education.

The U.S. National Geodetic Survey (NGS) developed the SP3 format, which later became the international standard (Yahya, 2007). A precise ephemeris file in the SP3 format consists of two sections: a header and data.

Table (1). The satellites positions from IGS web site and observed pseudoranges from observation massage of receiver for one epoch at 13:30:00 (26-9-2013).

\begin{tabular}{|c|c|c|c|c|}
\hline \multirow{2}{*}{ Satellite } & \multicolumn{3}{|c|}{ Satellites positions } & $\begin{array}{c}\text { Observed } \\
\text { pseudoran } \\
\text { ges (m) }\end{array}$ \\
\cline { 2 - 4 } & $\mathbf{X}(\mathbf{m})$ & $\mathbf{Y}(\mathbf{m})$ & $\mathbf{Z}(\mathbf{m})$ & 21637418.24 \\
\hline PG03 & 15259595 & -4413013.7 & 20771996 & 2094092.74 \\
\hline PG06 & 17717404 & 3275375.9 & 19689187 & 23962343.7 \\
\hline PG14 & 14962.323 & 18314.571 & -12020.7 & 24101004.74 \\
\hline PG19 & 10840307 & -12696573. & 20439741 & 20319250.63 \\
\hline PG22 & 21142.58 & 11404.859 & 11689.311 & 21531291.02 \\
\hline PG27 & 15871363 & -744733.77 & 21273725 & 23891578.5 \\
\hline PG29 & 3247.3345 & 25066.053 & -8183.3 & 234158.2 \\
\hline PG30 & 10279.57 & 24406.665 & -3355.6 & 2325 \\
\hline
\end{tabular}


Doma M.I.and Sedeek A. T. " Estimation of GPS Position Using Iterative Least Squares and Extended.........."

Table (2). The estimated positions for the GPS point from iterative least squares (ILS) method and extended Kalman filter (EKF)

\begin{tabular}{|c|c|c|c|c|c|c|}
\hline \multirow{2}{*}{ Epochs } & \multicolumn{3}{|c|}{ Estimated positions from EKF } & \multicolumn{3}{|c|}{ Estimated positions from ILS } \\
\hline & $\mathbf{X}(\mathbf{m})$ & $\mathbf{Y}(\mathbf{m})$ & $\mathbf{Z}(\mathbf{m})$ & $\mathbf{X}(\mathbf{m})$ & $\mathbf{Y}(\mathbf{m})$ & $\mathbf{Z}(\mathbf{m})$ \\
\hline 1 & 4710458.84 & 2831483.23 & 3225788.46 & 4710450.96 & 2831465.8 & 3225783.6 \\
\hline 2 & 4710448.05 & 2831477.23 & 3225790.29 & 4710451.95 & 2831470.9 & 3225774.3 \\
\hline 3 & 4710447.15 & 2831469.68 & 3225788.38 & 4710450.45 & 2831466.17 & 3225785.88 \\
\hline 4 & 4710447.15 & 2831468.95 & 3225786.29 & 4710446.77 & 2831471.97 & 3225790.83 \\
\hline 5 & 4710450.54 & 2831463.73 & 3225783.89 & 4710451.81 & 2831461.95 & 3225785.90 \\
\hline 6 & 4710451.27 & 2831464.55 & 3225778.68 & 4710450.30 & 2831468.79 & 3225776.53 \\
\hline 7 & 4710452.75 & 2831463.80 & 3225776.94 & 4710452.03 & 2831463.70 & 3225783.03 \\
\hline 8 & 4710453.46 & 2831465.04 & 3225770.35 & 4710452.69 & 2831468.22 & 3225767.44 \\
\hline 9 & 4710452.91 & 2831465.34 & 3225765.91 & 4710452.20 & 2831466.51 & 3225765.83 \\
\hline 10 & 4710454.66 & 2831466.03 & 3225764.74 & 4710453.41 & 2831463.99 & 3225779.50 \\
\hline 11 & 4710455.87 & 2831466.62 & 3225764.75 & 4710454.51 & 2831464.19 & 3225778.83 \\
\hline 12 & 4710453.88 & 2831467.14 & 3225765.64 & 4710450.84 & 2831463.42 & 3225787.55 \\
\hline 13 & 4710454.19 & 2831466.76 & 3225767.57 & 4710452.58 & 2831462.09 & 3225788.07 \\
\hline 14 & 4710455.03 & 2831469.69 & 3225765.40 & 4710453.80 & 2831469.60 & 3225774.64 \\
\hline 15 & 4710452.90 & 2831470.13 & 3225762.72 & 4710451.39 & 2831468.41 & 3225770.65 \\
\hline 16 & 4710453.16 & 2831468.72 & 3225768.28 & 4710451.10 & 2831460.56 & 3225799.84 \\
\hline 17 & 4710453.23 & 2831466.52 & 3225771.67 & 4710451.49 & 2831459.77 & 3225795.83 \\
\hline 18 & 4710453.42 & 2831465.04 & 3225770.04 & 4710453.40 & 2831463.62 & 3225772.41 \\
\hline 19 & 4710453.45 & 2831463.48 & 3225773.38 & 4710451.63 & 2831458.22 & 3225795.70 \\
\hline 20 & 4710452.78 & 2831464.92 & 3225771.45 & 4710451.60 & 2831465.39 & 3225776.91 \\
\hline 21 & 4710453.43 & 2831465.21 & 3225771.56 & 4710452.45 & 2831462.87 & 3225783.66 \\
\hline 22 & 4710453.32 & 2831466.40 & 3225769.32 & 4710452.19 & 2831465.75 & 3225776.66 \\
\hline 23 & 4710453.17 & 2831467.16 & 3225767.83 & 4710453.04 & 2831466.98 & 3225768.78 \\
\hline 24 & 4710451.60 & 2831467.22 & 3225770.83 & 4710449.26 & 2831462.17 & 3225793.90 \\
\hline 25 & 4710451.49 & 2831468.03 & 3225770.19 & 4710450.96 & 2831466.70 & 3225776.97 \\
\hline
\end{tabular}

Table (2) shows the estimated positions for GPS point from iterative least squares method and the extended Kalman filter algorithm. Also, Figure (3) shows relative positioning error in $\mathrm{x}, \mathrm{y}$ and $\mathrm{z}$ for both EKF and ILS.

To measure the quality of anything an index is required, standard deviation of the estimated coordinates of the GPS point can be used as an index in the present study. From these results, one can calculate the mean value of the GPS point coordinates

Arithmetic mean $(\bar{x})=\frac{\sum_{i=1}^{n} x_{i}}{n}$ 
Where :

$\mathrm{x}_{\mathrm{i}}$ : the estimated position of GPS point $(\mathrm{X}$, Y or Z) and

$\mathrm{n}$ : The number of measurements

The mean values of the positioning results for the 25 measurements for both methods are computed, the deviations of the values of the position estimates from the mean coordinates are shown in Fig. 3. From these values, one can compute the standard error as:

$$
\sigma_{x}= \pm \sqrt{\frac{\sum_{1}^{n} x_{i}^{2}-n \cdot \bar{x}^{2}}{(n-1)}}
$$

Where :

$\boldsymbol{\sigma}_{\mathbf{X}}$ : the standard error of GPS point (X, Y or Z).

- For EKF, one can compute the standard error as: $\sigma_{X}=2.56 \mathrm{~m} \& \sigma_{Y}=4.33 \mathrm{~m} \& \sigma_{Z}=8.29 \mathrm{~m}$

$>$ Similarly, for ILS method, one can compute the standard error as:

$\sigma_{\mathrm{X}}=1.87 \mathrm{~m} \& \sigma_{\mathrm{Y}}=4.29 \mathrm{~m} \& \sigma_{\mathrm{Z}}=12.68 \mathrm{~m}$

Finally, the resultant of the standard error of GPS position components is:

$$
\sigma= \pm \sqrt{\sigma_{X}^{2}+\sigma_{Y}^{2}+\sigma_{Z}^{2}}
$$

* For EKF, one can compute the resultant of standard error of GPS components as:

$$
\sigma_{\text {EKF }}=9.693 \mathrm{~m}
$$

Similarly, for ILS method, one can compute the resultant of standard error of GPS components:

$$
\sigma_{\text {ILS }}=\mathbf{1 3 . 5 2} \mathrm{m}
$$

$\checkmark \quad$ From these results, one can shows that the EKF is more quality than ILS method

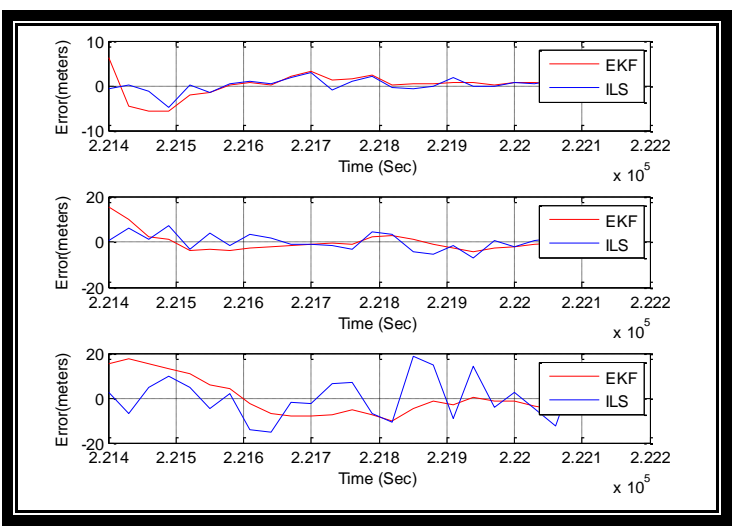

Figure 3. Relative positioning error in $\mathrm{x}, \mathrm{y}$ and $\mathrm{z}$ directions for Extended Kalman Filter (EKF) and Iterative Least Squares (ILS)

\section{Conclusions}

In this paper, we provide an insight in estimating the GPS receiver position using the single frequency L1 pseudorange measurements. Kalman filter is the method most often used nowadays. It is based on some assumptions and if all the assumptions are met it can offer optimal estimation and prediction. Also, the most commonly used algorithm for position computation from pseudoranges is based on the Least Square (LS) method. This method is used to find the receiver position from pseudoranges to four or more satellites.

In this study, In this paper, we are interested in estimating the receiver coordinates, combining all the information about noise, using iterative least square and extended Kalman filter. From the result of this study, EKF is more quality than ILS method

\section{References}

Battin, R. H., 1999, An Introduction to the Mathematics and Methods of Astrodynamics, Revised ed. Reston, VA: American Institute of Aeronautics and Astronautics, Inc.

Borre, K., Akos, D., Bertelsen, N., Rinder, P., and Jensen, S.: A Software Defined GPS and Galileo Receiver, Birkh“auser, 2007.

Chaer, W. S., Bishop, R. H. and Ghosh, J., 1997, A Mixture-of-Experts Framework for Adaptive Kalman Filtering", IEEE Transactions on Systems, Man and Cybernetics - Part B: Cybernetics, Vol.27, No.3, pp.452-464, 1997.

Götze, J.: Iterative Version of the QRD for Adaptive RLS Filtering, SPIE Conference on, Advanced Signal Processing: Algorithms, Architectures and Implementations, 438-450, 1994.

He, Y. and Bilgic, A., 2011, Iterative least squares method for global positioning system, Advances in Radio Science, 203-208, 2011.

Hofmann-Wellenhof, B., Lichtenegger, H., Collins, J.,1994, GPS Theory and Practice, Third Revised Edition, Springer-Verlag, New York, NY, 1994.

Hu, C. W.; Chen, W.; Chen, Y.; Liu, D., 2003, Adaptive Kalman Filtering for Vehicle Navigation, Journal of Global Positioning System, Vol. 2, No. 1, pp. 42-47.

Kalman R. E., "A new approach to linear filtering and prediction problems," Transactions of the ASME-Journal of Basic Engineering, pp. 35-45, March 1960.

Leick, A.,1995, GPS Satellite Surveying , Second Edition, John Wiley \& Sons, INC. 
Saeeas, I.; Gerakios, G.; Diamantis, A.; Dounis, A.I and Syrcos, G. P., 2010, Static Single Point Positioning Using the Extended Kalman Filter, International Journal of Information and Communication Engineering 6:3 2010

Simon, D., 2006, Optimal State Estimation: Kalman, 3TH. Infinity3T and Nonlinear Approches", John Wiley, 2006.

Spilker,J. J., Parkinson,B. W., Axelrad, P. and Enge, P., 1996, Global Positioning System: Theory and Application, Reston, VA: American Institute of Aeronautics and Astronautics, Inc., 1996, vol. I.
Xu, Benlin (1996), A New Navigation Filter. Ph.D. dissertation, Department of Geodesy and Geomatics Engineering, Technical Report No. 182, University of New Brunswick, Fredericton, New Brunswick, Canada, $111 \mathrm{pp}$.

Yahya.F., (2007), Accurate detection of small movement of L1 GPS antenna using digital signal processing techniques, M.sc. thesis under supervision of Helwan university, Cairo, Egypt. 